\title{
A cultura de segurança do paciente em um hospital privado no Sul do Brasil
}

\author{
The culture of patient safety in a private hospital in southern Brazil \\ La cultura de la seguridad del paciente en un hospital privado del Sul de Brasil
}

Recebido: 23/10/2021 | Revisado: 31/10/2021 | Aceito: 01/11/2021 | Publicado: 03/11/2021

Lilian Valesca Marinho Florencio ORCID: https://orcid.org/0000-0001-7675-4851

Universidade de Cruz Alta, Brasil

E-mail: 1ylyanflorencio@gmail.com

Éder Luís Arboit

ORCID: https://orcid.org/0000-0001-8929-5228

Universidade de Cruz Alta, Brasil

E-mail: earboit@unicruz.edu.br

Maria Cristina Schettert Moraes

ORCID: https://orcid.org/0000-0001-7666-2711

Universidade de Cruz Alta, Brasil

E-mail: mmoraes@unicruz.edu.br

Luís Felipe Pissaia

ORCID: https://orcid.org/0000-0002-4903-0775

Universidade do Vale do Taquari, Brasil

E-mail: lpissaia@universo.univates.br

\begin{abstract}
Resumo
Objetivo: Analisar a cultura de segurança do paciente em um hospital privado no Sul do Brasil. Método: Trata-se de um estudo exploratório, descritivo de abordagem quantitativa, realizada em agosto e setembro de 2019 por meio do Safety Attitudes Questionnaire. Participaram 100 trabalhadores de diversas categorias profissionais e lotados nos três turnos de trabalho. A análise dos dados foi realizada por meio da estatística descritiva. Resultados: A cultura de segurança do paciente na instituição é considerada adequada, uma vez que a maioria dos domínios apresentou um percentual entre $50 \%$ e $75 \%$ de positividade. Conclusões: Considera-se os resultados positivos, uma vez que os resultados apresentam-se como positivos para a maioria dos participantes da pesquisa, contudo é necessário investir na qualificação das práticas em saúde, principalmente por meio da educação continuada e permanente das equipes multiprofissionais atuantes e disponibilização de ferramentas que auxiliem nessas dinâmicas.
\end{abstract}

Palavras-chave: Segurança do paciente; Equipe multiprofissional; Cultura organizacional.

\begin{abstract}
Objective: To analyze the patient safety culture in a private hospital in southern Brazil. Method: This is an exploratory, descriptive study with a quantitative approach, carried out in August and September 2019 through the Safety Attitudes Questionnaire. 100 workers from different professional categories participated in the three work shifts. Data analysis was performed using descriptive statistics. Results: The patient safety culture in the institution is considered adequate, since most domains presented a percentage between $50 \%$ and $75 \%$ of positivity. Conclusions: The results are considered positive, since the results are positive for most research participants, however it is necessary to invest in the qualification of health practices, mainly through the continuous and permanent education of the active multidisciplinary teams and availability of tools that help in these dynamics.
\end{abstract}

Keywords: Patient safety; Multiprofessional team; Organizational culture.

\section{Resumen}

Objetivo: Analizar la cultura de seguridad del paciente en un hospital privado del Sul de Brasil. Método: Se trata de un estudio exploratorio, descriptivo con enfoque cuantitativo, realizado en agosto y septiembre de 2019 a través del Cuestionario de Actitudes de Seguridad. En los três turnos de trabajo participaron 100 trabajadores de diferentes categorías profesionales. El análisis de los datos se realizó mediante estadística descriptiva. Resultados: La cultura de seguridad del paciente en la institución se considera adecuada, ya que la mayoría de los dominios presentaron un porcentaje entre $50 \%$ y $75 \%$ de positividad. Conclusiones: Los resultados se consideran positivos, ya que los resultados son positivos para la mayoría de los participantes de la investigación, sin embargo es necesario invertir en la calificación de las prácticas de salud, principalmente a través de la educación continua y permanente de los equipos multidisciplinarios activos y la disponibilidad de herramientas que ayuden en estas dinámicas.

Palabras clave: Seguridad del paciente; Equipo multiprofesional; Cultura de la organización. 


\section{Introdução}

A segurança do paciente é um tema debatido por muitas décadas, que por meio de outras nomenclaturas buscavam a qualificação dos serviços de saúde por meio de práticas principalmente assistenciais que visem a atender as demandas da população atendida, conforme indicam Santos et al. (2021). Conforme Harada et al. (2021) e Nora e Junges (2021) a qualificação dos serviços de saúde também buscam atingir os diversos processos de acreditação exigidos pelas organizações nacionais e internacionais de fiscalização e garantia dos direitos de acesso em saúde pela população.

Dessa forma, para Penariol et al. (2021) o conceito de segurança do paciente está ligado diretamente a capacidade do serviço de saúde em evitar lesões e danos à população atendida em meio às práticas de cuidado empregadas para a prevenção, tratamento ou reabilitação do indivíduo. Assim é possível compreender a amplitude que a segurança do paciente recebe em meio às políticas públicas nos serviços de saúde, qualificando os modelos de trabalho e oferta de procedimentos para a população com foco na resolutidade dos casos, conforme mencionam Chauhan, Kaur e Chang (2021).

Segundo Lima Neto et al. (2021) a importância da segurança do paciente começa com o processo de admissão do indivíduo no serviço de saúde, o foco principal desde o momento inicial é a preservação do bem-estar independente do caso, evitando os desconfortos relacionados a terapêutica empregada e evitando riscos assistenciais. Neste contexto, Alencar et al. (2021) mencionam que a prevenção de riscos está diretamente ligada a segurança do paciente, às quais podem aumentar o tempo de internação hospitalar e por consequente os custos assistenciais ligados a situação de saúde inicial, além de causar danos temporários e/ou permanentes no paciente atendido.

No que tange o erro profissional, Santos et al. (2021) consideram a capacitação profissional como o fator linear de prevenção destas situações, com foco na comunicação assertiva, direta e objetiva entre os membros da equipe multiprofissional e desta com os seus pacientes e familiares. Para Nora e Junges (2021) a capacitação da equipe multiprofissional começa desde a formação na área da saúde, seja em cursos de graduação, técnicos ou profissionalizantes e adaptados ao serviço de saúde por meio de ações estratégicas de educação continuada e permanente que são desenvolvidas pela gestão como maneira de qualificar os processos de trabalho no local.

Deste modo, evitar acidentes e lesões faz parte da segurança do paciente e ações preventivas devem ser abordadas com afinco nos serviços de saúde, compondo os meios de defesa dos direitos da população e cumprimento com os deveres éticos e legais da equipe de saúde, conforme indicam Siqueira et al. (2021) e Rocha et al. (2021). Tais estratégias são pensadas em consonância com o desenvolvimento da cultura de segurança do paciente, a qual estrutura-se em bases científicas das quais é possível identificar situações problema nos processos de trabalho, citam Torrente e Barbosa (2021).

A equipe multiprofissional está envolvida nas práticas de segurança do paciente, segundo Lima et al. (2021) a redução de riscos relacionados a assistência em saúde é um dever ético de todos os profissionais da saúde, com ênfase na preservação da vida e a integridade do organismo. Em meio ao processo de trabalho, algumas ações de segurança do paciente podem ser implementadas por meio da Comissão de Segurança do Paciente, grupo de profissionais capacitados por meio das políticas públicas vigentes e que desenvolvem um olhar holístico sobre os fluxos de trabalho em saúde de maneira crítica e reflexiva, segundo Alencar et al. (2021) comentam.

A implementação de ações de segurança do paciente seguem um limiar estipulado por metas internacionais, conforme mencionam Nunes (2021) e Barbosa et al. (2021) sendo a primeira a identificação do paciente, a segunda na prevenção de quedas, a terceira pactua sobre a higiene das mãos, seguida pelo viés de segurança medicamentosa e por fim a prevenção de lesões por pressão e cirurgia segura. Para Lima Neto et al. (2021) todas as metas traçadas facilitam a prevenção de riscos e fortalecem a vinculação de boas práticas em saúde com a população.

Ainda, a segurança do paciente não é somente dever do serviço de saúde ou dos profissionais da equipe multiprofissional, mas também do paciente, familiares e da população conforme indicam Carneiro et al. (2021) e Madalozzo et 
al. (2021). A comunidade auxilia o serviço de saúde e os profissionais por meio da identificação de situações problema que são passiveis de melhoria, além de estruturar boas práticas na comunicação entre paciente e equipe colaborando para o tratamento e acompanhamento do caso, segundo Mendes et al. (2021).

Desta forma, este estudo configura-se por entre o objetivo de analisar a cultura de segurança do paciente em um hospital privado no Sul do Brasil.

\section{Metodologia}

Trata-se de um estudo de abordagem quantitativa, descritivo e exploratório (Lopes, 2018), realizado em um hospital de caráter privado no Sul do Brasil. A população do estudo foi de 100 profissionais e a amostra foi composta por trabalhadores que atenderam aos seguintes critérios de inclusão: ser profissional enfermeiro, técnico de enfermagem, fisioterapeutas, nutricionistas, farmacêuticos, médicos, colaboradores do setor administrativo, copa, lavanderia, manutenção, higienização entre outros que trabalhem há pelo menos seis meses na instituição. Foram excluídos os profissionais que se encontravam de férias, licença de saúde ou qualquer outro afastamento no período de produção dos dados.

A coleta de dados foi realizada em agosto e setembro de 2019 por meio do Safety Attitudes Questionnaire, (SAQ) validado e adaptado culturalmente para a realidade brasileira por Carvalho (2011). O instrumento é composto 41 itens totais, subdividido em duas partes. A primeira parte compreende 36 itens distribuídos em seis domínios, conforme os dados do Quadro 1, apresentado abaixo.

Quadro 1. Domínios e definições do Safety Attitudes Questionnaire (SAQ). Cruz Alta, RS, 2021.

\begin{tabular}{|c|l|}
\hline Domínios & \multicolumn{1}{c|}{ Definição } \\
\hline $\begin{array}{c}\text { Clima de Trabalho em } \\
\text { equipe }\end{array}$ & Compreende a qualidade do relacionamento e a colaboração entre os membros da equipe. \\
\hline Clima de Segurança & $\begin{array}{l}\text { Avalia a percepção dos profissionais quanto ao comprometimento organizacional para segurança } \\
\text { do paciente. }\end{array}$ \\
\hline Satisfação no Trabalho & É a visão positiva do local de trabalho. \\
\hline Percepção de estresse & Trata do reconhecimento do quanto os fatores estressores influenciam na execução do trabalho. \\
\hline $\begin{array}{c}\text { Percepção da gerência da Unidade e do } \\
\text { Hospital }\end{array}$ & Refere-se a aprovação das ações da gerência quanto as questões de segurança. \\
\hline Condições de Trabalho & Refere-se a percepção da qualidade do ambiente de trabalho. \\
\hline
\end{tabular}

Fonte: Carvalho (2011).

A pontuação final do instrumento varia de 0 a 100. A pontuação é ordenada da seguinte forma: discordo totalmente (A) é igual a 0 pontos; discordo parcial (B) é igual a 25 pontos; indiferente (C) é igual a 50 pontos; concordo parcialmente (D) é igual a 75 pontos; e concordo totalmente (E) é igual a 100 pontos.

A partir desses valores, as respostas de cada domínio foram somadas e divididas pelo número de questões, resultando em uma variação de zero a 100. Os valores são considerados positivos quando a pontuação total é maior ou igual a 75. Assim, quanto maior o escore, mais positiva a atitude, com exceção dos itens que apresentam escore reverso $(2,11$ e 36) onde o menor escore indica uma atitude mais positiva (Carvalho, 2011).

O instrumento impresso foi entregue aos participantes para ser respondido e devolvido posteriormente, com data de devolução previamente definida pelos pesquisadores (retornando em sete dias). O projeto foi aprovado por Comitê de Ética em Pesquisa, sob o parecer $n^{\circ}$ 3.482.389. Ressalta-se que após a concordância em participar da pesquisa, os participantes foram convidados a ler e assinar o Termo de Consentimento Livre Esclarecido (TCLE) em duas vias de igual teor, ficando uma via 
com o pesquisador e outra via com o participante. Manteve-se a equivalência de representatividade entre as categorias profissionais e entre os diferentes turnos de trabalho.

Ao serem recolhidos os questionários, estes foram numerados com o código relativo à letra inicial da categoria profissional e sequencialmente numerados. Primeiramente, os resultados integraram um banco de dados em uma planilha do programa Excel $^{\circledR}$ for Windows ${ }^{\circledR} 2016$ e após analisados por meio da estatística descritiva, analítica e inferencial.

\section{Resultados}

Participaram do estudo 100 trabalhadores, sendo que 54 são membros da equipe de enfermagem. A maioria mulheres, com idade entre 30 a 40 anos e com tempo de atuação entre cinco a 10 anos. A Tabela 1, a qual está reservada aos dados profissionais, identificando o seu perfil (cargo, gênero, atuação principal e tempo de experiência na especialidade).

Tabela 1. Categoria profissional, sexo, idade e tempo de trabalho. Cruz Alta, RS, 2021.

\begin{tabular}{|c|c|c|}
\hline Categoria profissional & $\mathbf{N}$ & $\%$ \\
\hline Enfermeiro & 14 & $14 \%$ \\
\hline Farmacêutico & 03 & $3 \%$ \\
\hline Fisioterapeuta & 03 & $3 \%$ \\
\hline Nutricionista & 03 & $3 \%$ \\
\hline Psicóloga & 01 & $1 \%$ \\
\hline Biomédica & 01 & $1 \%$ \\
\hline Médico & 06 & $6 \%$ \\
\hline Técnico em Enfermagem & 40 & $40 \%$ \\
\hline Técnico em Radiologia & 04 & $4 \%$ \\
\hline Técnico em informática & 01 & $1 \%$ \\
\hline Auxiliar Administrativo & 09 & $9 \%$ \\
\hline Auxiliar de farmácia & 03 & $3 \%$ \\
\hline Copa / cozinha & 03 & $3 \%$ \\
\hline Higienização & 04 & $4 \%$ \\
\hline Lavanderia & 02 & $2 \%$ \\
\hline Manutenção & 01 & $1 \%$ \\
\hline Maqueiro & 01 & $1 \%$ \\
\hline Cuidadora & 01 & $1 \%$ \\
\hline \multicolumn{3}{|l|}{ Sexo } \\
\hline Masculino & 19 & $19 \%$ \\
\hline Feminino & 81 & $81 \%$ \\
\hline \multicolumn{3}{|l|}{ Idade } \\
\hline 20 a 30 anos & 27 & $27 \%$ \\
\hline 30 a 40 anos & 45 & $45 \%$ \\
\hline 40 a 50 anos & 16 & $16 \%$ \\
\hline 50 a 60 anos & 11 & $11 \%$ \\
\hline 60 a 70 anos & 01 & $1 \%$ \\
\hline \multicolumn{3}{|l|}{ Tempo de trabalho } \\
\hline 6 a 11 meses & 10 & $10 \%$ \\
\hline 1 a 2 anos & 08 & $8 \%$ \\
\hline 3 a 4 anos & 19 & $19 \%$ \\
\hline 5 a 10 anos & 37 & $37 \%$ \\
\hline 11 a 20 anos & 21 & $21 \%$ \\
\hline 21 ou mais & 05 & $5 \%$ \\
\hline
\end{tabular}

Fonte: Autores (2021).

A Tabela 2 está constituída em seis domínios: clima de trabalho e satisfação no trabalho com $76 \%$ e $85 \%$ o que caracteriza um percentual ‘forte’ de positividade. Os domínios clima de segurança, percepção de stress, percepção da gerência 
e condições de trabalho obtiveram resultados de 67\%, 63\%, 54\% e 59\% respectivamente, sendo possível evidenciar uma cultura 'adequada' de segurança do paciente.

Tabela 2. Domínios do Safety Attitudes Questionnaire (SAQ) em um hospital privado do interior gaúcho. Cruz Alta, RS, 2021.

\begin{tabular}{|c|c|}
\hline Questões por domínio & Positividade \\
\hline \multicolumn{2}{|l|}{ Clima de trabalho em equipe } \\
\hline $\begin{array}{l}\text { 1. As sugestões dos enfermeiros são bem recebidas neste serviço. } \\
\text { 2. Neste serviço é difícil falar livremente se me aperceber de um problema na prestação de cuidados ao doente. } \\
\text { 3. As divergências neste serviço são resolvidas adequadamente. } \\
\text { 4. Tenho o apoio de que necessito por parte de outros profissionais na prestação de cuidados aos doentes. } \\
\text { 5. É fácil para os profissionais que atuam neste serviço colocarem questões quando não compreendem algo. } \\
\text { 6. Os médicos(as) e enfermeiros(as) deste serviço trabalham em conjunto como uma equipe bem coordenada. } \\
\text { Percentual de positividade/classificação }\end{array}$ & $\begin{array}{r}84 \% \\
49 \% \\
79 \% \\
84 \% \\
81 \% \\
79 \% \\
76 \% \text { (forte) } \\
\end{array}$ \\
\hline \multicolumn{2}{|l|}{ Clima de segurança } \\
\hline $\begin{array}{l}\text { 7. Sentir-me-ia seguro caso fosse tratado aqui como doente. } \\
\text { 8. Os erros dos profissionais são tratados de forma adequada neste serviço. } \\
\text { 9. Conheço os meios adequados para encaminhar questões relacionadas com a segurança do doente neste serviço. } \\
\text { 10. Recebo feedback adequado acerca do meu desempenho. } \\
\text { 11. Neste serviço é difícil discutir os erros. } \\
\text { 12. Sou encorajado pelos meus colegas a reportar qualquer preocupação que possa a ter com a segurança do doente. } \\
\text { 13. A cultura neste serviço faz com que seja fácil aprender com os erros dos outros. } \\
\text { Percentual de positividade/classificação }\end{array}$ & \begin{tabular}{r|}
$83 \%$ \\
$75 \%$ \\
$83 \%$ \\
$51 \%$ \\
$44 \%$ \\
$76 \%$ \\
$60 \%$ \\
$67 \%$ (Adequado) \\
\end{tabular} \\
\hline \multicolumn{2}{|l|}{ Satisfação no trabalho } \\
\hline $\begin{array}{l}\text { 15. Gosto do meu trabalho. } \\
\text { 16. Trabalhar aqui é como fazer parte de uma grande família. } \\
\text { 17. Este é um bom local para trabalhar. } \\
\text { 18. Tenho orgulho em trabalhar neste serviço. } \\
\text { 19. A moral neste serviço é alta. } \\
\text { Percentual de positividade/classificação }\end{array}$ & $\begin{array}{r}95 \% \\
82 \% \\
91 \% \\
90 \% \\
71 \% \\
85 \% \text { (Forte) } \\
\end{array}$ \\
\hline \multicolumn{2}{|l|}{ Percepção de stress } \\
\hline $\begin{array}{l}\text { 20. Quando a minha carga de trabalho se torna excessiva, o meu desempenho é prejudicado. } \\
\text { 21. Sou menos eficiente no trabalho quando estou cansado. } \\
\text { 22. Tenho maior probabilidade de cometer erros em situações tensas ou hostis. } \\
\text { 23. A fadiga influencia o meu desempenho em situações de emergência. } \\
\text { Percentual de positividade/classificação }\end{array}$ & $\begin{array}{c}80 \% \\
76 \% \\
58 \% \\
41 \% \\
63 \% \text { (Adequado) } \\
\end{array}$ \\
\hline \multicolumn{2}{|l|}{ Percepção da gerência } \\
\hline $\begin{array}{l}\text { 24. A Administração apoia os meus esforços diários. } \\
\text { 25. A Administração não compromete de forma consciente a segurança do doente. } \\
\text { 26. A Administração está a realizar um bom trabalho. } \\
\text { 27. Profissionais problemáticos são tratados de forma construtiva. } \\
\text { 28. Recebo informações adequadas e oportunas sobre eventos que podem afetar o meu trabalho. } \\
\text { 29. O número de profissionais deste serviço é suficiente para tratar o número de doentes. } \\
\text { Percentual de positividade/classificação }\end{array}$ & $\begin{array}{r}57 \% \\
51 \% \\
65 \% \\
58 \% \\
65 \% \\
32 \% \\
54 \% \text { (Adequado) } \\
\end{array}$ \\
\hline \multicolumn{2}{|l|}{ Condições de trabalho } \\
\hline $\begin{array}{l}\text { 30. Este hospital realiza um bom trabalho na formação de novos profissionais. } \\
\text { 31. Toda a informação necessária para as decisôes diagnósticas e terapêuticas é-me disponibilizada de forma } \\
\text { sistemática. } \\
\text { 32. Os estágios da minha profissão são adequadamente supervisionados. } \\
\text { Percentual de positividade/classificação }\end{array}$ & $\begin{array}{l}65 \% \\
58 \% \\
54 \% \\
59 \% \text { (Adequado) } \\
\end{array}$ \\
\hline
\end{tabular}

Fonte: Autores (2021).

A tabela número três, apresenta questões que não foram contempladas nos domínios do SAQ, sendo questões referentes a sugestões, vivências e falhas de comunicação, as quais obtiveram um percentual de 66\%. Esse valor é considerado adequado. 
Tabela 3. Análise descritiva de outros itens do SAQ que não estão contemplados nos domínios. Cruz Alta - RS, Brasil, 2021.

\begin{tabular}{|c|c|}
\hline Questões por domínio & Positividade \\
\hline Outros itens do SAQ que não estão contemplados nos domínios & \\
\hline $\begin{array}{l}\text { 14. As minhas sugestões acerca da segurança seriam postas em prática caso as expressasse à administração. } \\
\text { 33. Vivencio boa colaboração com os(as) enfermeiros(as) neste serviço. } \\
\text { 34. Vivencio boa colaboração com os(as) médicos(as) neste serviço. } \\
\text { 35. Vivencio boa colaboração com os(as) farmacêutico(as) neste serviço. } \\
\text { 36. São comuns falhas de comunicação que levam a atrasos na prestação de cuidados. } \\
\text { Percentual de positividade/classificação }\end{array}$ & $\begin{array}{c}61 \% \\
89 \% \\
82 \% \\
84 \% \\
21 \% \\
67 \% \text { Adequado) }\end{array}$ \\
\hline
\end{tabular}

Fonte: Autores (2021).

A seguir, serão apresentadas as discussões pertinentes aos resultados da pesquisa.

\section{Discussões}

Esta seção apresenta ao leitor as discussões pertinentes ao estudo, correlacionando os resultados da pesquisa apresentados na seção anterior com a bibliografia científica disponível na atualidade. A segurança do paciente enquanto prática profissional na área da saúde recebeu destaque somente na última década, conforme Viana et al. (2021) e Carneiro et al. (2021) autores que incitam sobre a implementação de políticas públicas que implementam e auxiliam na indicação da determinada prática.

Dessa forma, a segurança do paciente da forma como conhecemos é uma construção histórica para a área da saúde, vinda principalmente ao encontro da necessidade de certificação dos serviços de saúde perante a qualidade dos atendimentos oferecidos para a população, conforme indicam Rocha et al. (2021). Ao passo que tais práticas se relacionam diretamente com preceitos por vezes "obrigatórios" para a adequação da instituição, os profissionais da equipe de saúde não compreendem o seu objetivo e o processo de implementação das boas práticas internacionais, segundo observa o estudo de Barbosa et al. (2021).

Neste contexto, ao analisar os domínios do SAQ, foi possível classificar o índice de positividade da cultura de segurança do paciente como forte e adequado. O achado vai ao encontro com os estudos realizados por Ruth et al. (2021) e Rocha et al. (2021) em que a cultura de segurança do paciente é verificada como fruto de um longo processo de conscientização das equipes de saúde, tornando-se educativo ao passo que exige práticas de pesquisa in loco, implementação de cronogramas de educação continuada e permanente para o desenvolvimento profissional, bem como a identificação de situações problema à beira do leito e que podem ser solucionadas. Vale ressaltar que o resultado observado indica que aqueles domínios com 75\% ou mais de concordância são "fortes". Considera-se "adequado" as respostas com percentual entre 50 a $74.9 \%$ e foram consideradas como "frágil" as dimensões em que as respostas positivas ficaram abaixo de $50 \%$, o que indica a necessidade de intervenções para melhoria da segurança do paciente.

Para Nora e Junges (2021) os profissionais da saúde que estão imersos em práticas assistenciais, aquelas denominadas a beira do leito, estão mais propensas a desenvolver práticas errôneas ou consonantes com negligencias relacionadas às práticas de segurança do paciente, seja pela falta de conhecimento sobre a área de atuação ou falta de documentos, protocolos e rotinas de direcionamento do cuidado prestado naquele local. Assim, perceber-se em situações de negligência para com a segurança do paciente é o primeiro passo para a correção e direcionamento de uma boa prática que conforme Nunes (2021) e Siqueira et al. (2021) demandam um bom relacionamento entre os componentes da equipe, uma gestão eficaz e consciência do seu papel perante às necessidades da população e do dever ético que cerca o profissional da saúde.

Ainda assim, a cultura de segurança do paciente é um campo delicado de ser trabalhado, ao passo que a pesquisa realizada por Penariol et al. (2021) indica a ineficácia dos dados de oriundos de notificações relacionadas às faltas no campo assistencial. Para Mendes et al. (2021) a subnotificação de agravos ou eventos relacionados a assistência em saúde é fruto da 
desinformação em que o tema de segurança do paciente esta imerso, falta ética ao omitir o erro e também a postura do serviço de saúde que realiza punições aos profissionais que cometem este tipo de erro.

Para este contexto, Torrente e Barbosa (2021) indicam a formulação de estratégias de cultura de segurança do paciente, sobretudo aquelas fundamentadas cientificamente e que são fundamentais para o campo assistencial, como por exemplo, a implementação de rotinas profissionais que descrevem as boas práticas para cada especialidade, constituindo-se em suma como as atribuições do enfermeiro, técnico em enfermagem, médico, por exemplo. Segundo Lima et al. (2021) pequenas mudanças na rotina profissional interferem positivamente para o desenvolvimento da segurança do paciente, auxiliando os profissionais a refletirem sobre a própria prática e encontrar o limiar de atuação saudável e condizente com a necessidade do serviço de saúde e da população.

Outra estratégia eficaz para a sensibilização em segurança do paciente é a educação continuada e permanente em saúde que conforme Oliveira e Toledo (2021) buscam incialmente sensibilizar os profissionais da equipe multiprofissional, fazendo-o refletir sobre as ações e processos de trabalho, abrindo espaço para a mudança. Alencar et al. (2021) comentam sobre os resultados positivos de treinamentos e momentos educativos em um a instituição de saúde, sendo possível capacitar os profissionais e estes ficam responsáveis por disseminar as informações para os pacientes, principalmente quando o indivíduo necessita de cuidados especiais, sejam cuidadores formais ou informais, essas pessoas são a extensão do cuidado em sua essência profissional.

As discussões tornam-se pertinentes ao verificar que no domínio responsável por avaliar o clima de trabalho em equipe, observou-se um percentual de $76 \%$, de positividade o que demonstra um bom incentivo e qualificação já existente neste local. Tal achado está amparado nas construções de Jost et al. (2021) os quais mencionam a melhoria na segurança do paciente a medida que o relacionamento entre a equipe se fortalece, criando uma rotina de momentos de reflexão, capacitações sobre diversos temas e conexão mutua entre as pessoas.

Contudo, o mesmo questionário identificou que se pode melhorar ainda mais para que os trabalhadores sintam-se realmente amparados neste espaço, pois ao observar o item dois do domínio clima de trabalho, a metade dos participantes, ou seja, 50\% demonstram enfrentar dificuldades, não se sentem a vontade ao relatar os problemas livremente dentro da instituição em que trabalham. No estudo de Santos et al. (2021) a dificuldade sobre expor os erros relacionados a assistência em saúde está ligado com o perfil de gerenciamento do serviço de saúde, o qual influencia diretamente nos relacionamentos interpessoais entre a equipe multiprofissional. Da mesma forma, Harada et al. (2021) pactuam sobre a necessidade dos gestores dos serviços e saúde acolherem as equipes de maneira que o erro seja compreendido em seu contexto, trabalhando estratégias de prevenção e resolução quando aconteça, tirando o foco do modelo punitivo e que encontre os dados reais sobre o erro profissional.

A segurança do paciente é influenciada diretamente pelas relações que a equipe possui entre si, principalmente na capacidade de refletir sobre as práticas em comum para a constante qualificação do serviço de saúde, conforme Siqueira et al. (2021) e Ruth et al. (2021) comentam. Consonante a isso, no item um e quatro (domínio um) evidencia-se um percentual de $84 \%$, considerado forte, demonstrando que as maiorias dos colaboradores sentem-se satisfeitos com as sugestões recebidas pelos enfermeiros e pelo apoio dos demais profissionais. Tal informação está de acordo com a pesquisa realizada por Alencar et al. (2021) e que relaciona a qualidade da assistência com o bem-estar da equipe multiprofissional, pactuando sobre o espirito de cooperação e sobretudo as trocas entre áreas desenvolvidas nos momentos de educação continuada e permanente.

Ainda sobre a satisfação dos participantes quanto ao ambiente de trabalho, Oliveira e Toledo (2021) trazem à tona a problemática dos ambientes sem a segurança profissional necessária para atuação da equipe de enfermagem, situações como a falta de Equipamentos de Proteção Individual (EPIs) e ferramentas tecnológicas que auxiliem nas demandas assistenciais e gerenciamento do serviço, se revelam como propensos para a ocorrência de faltas à segurança do paciente. Sob o mesmo limiar, Lima Neto et al. (2021) colaboram com o contexto indicando que a satisfação com o ambiente de trabalho confere o 
bem-estar necessário para o profissional e a equipe desenvolver as suas atividades com atenção, cuidado e zelo perante às necessidades da população, além de respeitar os preceitos éticos e humanístico.

Em relação ao domínio dois, o qual mensurou o clima de segurança, observou-se um percentual adequado de positividade de $67 \%$. Este limiar de positividade é relacionado com a abertura para desenvolver a cultura de segurança do paciente e ainda um olhar aberto e flexível sobre as demandas do serviço de saúde, conforme comentam Silva et al. (2021). A positividade forte também se relaciona com a abertura das orientações recebidas pelos membros da equipe de saúde, o que de fato é um diferencial para o desenvolvimento das práticas de segurança do paciente, conforme indicam Madalozzo et al. (2021).

Contudo, evidencia-se que no item 11 os participantes indicaram uma fragilidade de $44 \%$, pois os trabalhadores afirmaram que neste serviço é difícil de discutir os erros quando ocorridos, uma vez que pode haver sobre carga de trabalho ou pressão na realização das atividades, não havendo assim tempo disponível para a discussão dos erros. Viana et al. (2021) e Carneiro et al. (2021) mencionam em suas pesquisas sobre a necessidade de avaliar o tempo disponível para as equipes desenvolverem boas práticas relacionadas a segurança do paciente, sem contar os treinamentos e capacitações que acontecem normalmente no serviço de saúde. Ainda, Rocha et al. (2021) alerta os gestores dos serviços de saúde para a sobrecarga de trabalho, pontuando a perda da qualidade assistencial por motivos de cansaço, desmotivação ou julgamento da equipe multiprofissional. Da mesma forma, Nunes (2021) e Torrente e Barbosa (2021) indagam sobre os casos de erro profissional relacionado ao cansaço e dupla jornada de trabalho, que causam delitos éticos principalmente de imperícia.

Já no item sete os profissionais expressaram sua segurança caso fossem pacientes nesta instituição, pois 83\% demonstraram confiança neste local para uma futura internação hospitalar. Quando avaliado a percepção dos profissionais perante a própria assistência, é possível identificar o esforço com que as equipes conduzem a segurança do paciente enquanto premissa do contexto laboral, conforme Maladozzo et al. (2021) comentam. Dessa forma, ao colocar-se empaticamente no lugar do seu próprio paciente, o profissional reflete sobre a prática que desenvolve rotineiramente, aproximando as dores e as possibilidades de qualificação da assistência segundo Lima Neto et al. (2021). Para Nunes (2021) confiar no próprio local de trabalho é sentir-se seguro para desenvolver as atividades, é compartilhar da inquietude quanto a qualificação do modelo assistencial realizado pelo serviço e, sobretudo, respeitar as individualidades e o perfil do serviço de saúde das equipes.

Ainda sobre a qualidade de vida no trabalho, no domínio três satisfação laboral, as perguntas de número 15 á 19 apresentaram percentuais de $71 \%$ a $95 \%$, pois os trabalhadores demonstraram-se satisfeitos e orgulhosos em atuar nesta instituição hospitalar. Compreende-se que gostar do trabalho que desenvolve é um dos pilares para o bem-estar profissional, que interfere diretamente na qualidade de vida dos profissionais e na maneira como ele se percebe no contexto da empresa em que trabalha, conforme reflexões realizadas por Harada et al. (2021).

Para Chauhan, Kaur e Chang (2021) o serviço de saúde compreende que as atitudes positivas dos profissionais colaboram com a qualidade dos serviços prestados para a população, incorporando valores como a humanização e a empatia perante as situações que acometem os indivíduos em acompanhamento pela equipe. Dessa forma, trabalhar em prol da equipe multiprofissional é também qualificar o serviço de saúde por meio das entregas à população atendida, dentre elas a segurança do paciente, responsabilidade social e sustentabilidade do negócio, conforme citam Siqueira et al. (2021).

No domínio quatro, avaliando a percepção do stress dos trabalhadores da instituição pesquisada, obtivemos um percentual de 63\% considerado adequado, o item 20 apresenta o contentamento dos colaboradores em relação as suas respectivas funções, pois $80 \%$ deles afirmam não serem prejudicados quando há excesso de trabalho. Esta é uma questão muito pessoal que varia de colaborador para colaborador, cada ser humano reage de maneira diferente a qualquer situação e só cabe a si próprio avaliar o que lhe estressa ou não. Dessa forma, Santos et al. (2021) e Oliveira e Toledo (2021) comentam que a carga de trabalho pode interferir no clima da organização e consequentemente no clima de segurança, sendo que profissionais 
sobrecarregados possuem a predisposição de terem uma percepção ruim quanto ao clima de trabalho em equipe, quanto à gerência da unidade e do hospital, condições de trabalho, satisfação laboral, percepção do estresse e no clima de segurança.

O domínio cinco apresenta-se adequado com um percentual de 54\% de positividade na percepção dos colaboradores em relação á gerência da instituição. Nos estudos de Lima et al. (2021) e Viana et al. (2021) a fragilidade gerencial é um dos principais fatores determinantes no sucesso ou insucesso de implementação de projetos que envolvam a segurança do paciente, sobretudo aqueles que demandem o envolvimento da equipe na construção e elaboração de ações. Entende-se que a gestão é o principal motivador para o desenvolvimento de ações em segurança do paciente, qualificando as práticas de educação continuada e permanente, além de estruturar um espaço de criação e inovação em saúde.

No item 29, obteve-se uma insatisfação de $32 \%$ dos colaboradores a respeito da insuficiência do número adequado de profissionais em relação á quantidade elevada de doentes a serem atendidos. Essa informação torna-se preocupante ao revelar um dificuldade na implementação de ações que favoreçam as práticas de segurança do paciente, incluindo as medidas de prevenção aos erros assistenciais ligados ao excesso de trabalho da equipe. Para Madalozzo et al. (2021) o dimensionamento das equipes de saúde, com foco na de enfermagem é essencial para o sucesso da assistência oferecida para os pacientes, de modo que os recursos profissionais sejam suficientes para amparar as situações problema presente no serviço de saúde. Da mesma forma, Harada et al. (2021) e Siqueira et al. (2021) abordam o dimensionamento das equipes de saúde como uma tarefa puramente gerencial sob o resguardo da legislação de exercício profissional de cada área, sendo necessário estruturar meios de atender a demanda real de profissional x pacientes em prol do sucesso dos serviços ofertados para a população.

No último domínio de número seis, em relação às condições de trabalho, os percentuais apresentam-se baixos, totalizando apenas 59\% de positividade, como demonstra o item 32 com escore de 54\%. Cabe ressaltar que no ano de 2019 não houve a oferta de estágios pela instituição pesquisada, portanto não colaborando com a formação de novos profissionais tanto na área da enfermagem como nas demais profissões. Considera-se que o caráter privado da instituição hospitalar pesquisada, determina a necessidade de parcerias e/ou prestação de serviços para as instituições de ensino como meio de fornecer espaço para as disciplinas práticas. Entende-se que a disponibilidade de estágios, disciplinas práticas ou espaço para capacitação de estudantes em parceria com a instituição é uma necessidade e principal saída para o desenvolvimento de uma formação adequada nos parâmetros estipulados pela legislação, conforme indica Jost et al. (2021).

A última tabela nos apresenta os itens que não foram inclusos nos 6 domínios acima, totalizando um percentual adequado de $66 \%$, demonstrando opiniões opostas, com percentuais elevados pois, no item $14,61 \%$ dos participantes da pesquisa acreditam que suas opiniões acerca da segurança seriam observadas e postas em prática caso as expressassem aos administradores da assistência. Pode-se observar que nesta instituição há um grande número de profissionais dispostos a colaborar junto da gerência, proporcionando aos pacientes uma assistência cada vez mais segura. Neste contexto, Mendes et al. (2021), Barbosa et al. (2021) e Rocha et al. (2021) reforçam a necessidade de construir um meio de trabalho com uma comunicação assertiva entre as áreas, principalmente os profissionais da saúde em conjunto com a gerencia do serviço de saúde, aproximando as ideias e otimizando as possibilidades de inovação no espaço de desenvolvimento das ações. Ainda para Alencar et al. (2021) a comunicação é um dos principais aspectos trabalhados no desenvolvimento da cultura de segurança do paciente, garantindo assim que as informações sejam discutidas, trabalhadas e utilizadas para a articulação de diferentes estratégias de enfrentamento das situações de crise. Nos itens 33, 34 e 35 os profissionais afirmam ter boa colaboração para com os enfermeiros totalizando $89 \%$. Já os médicos tem um percentual de $82 \%$ e farmacêuticos $84 \%$. Porém no último item de número 36 o percentual de positividade é baixo somente $17 \%$, confirmando que as falhas na comunicação levam ao atraso nos cuidados prestados aos pacientes. Percebe-se que estas informações colaboram com o achado anterior, demonstrando que a aproximação entre os profissionais e a comunicação assertiva induz a boas práticas assistências, construindo a cultura de segurança do paciente de uma maneira integral e resolutiva. 
Portanto as instituições devem cumprir o papel de incentivadores de seus funcionários, para que os mesmos não se sintam desmotivados ao exercerem suas funções no ambiente de trabalho, receber um elogio é muito bom, dignifica a pessoa e a motiva a ser um profissional cada vez melhor, além de favorecer as práticas de segurança do paciente indicadas neste estudo.

\section{Considerações Finais}

Compreende-se que este estudo cumpriu com o seu objetivo de analisar a cultura de segurança do paciente em um hospital privado no Sul do Brasil. O interesse pelo desenvolvimento deste estudo sobre a cultura de segurança do paciente, na perspectiva da equipe multiprofissional foi incentivado pela curiosidade em compreender a visão dos profissionais em relação ao hospital pesquisado, além de ser um tema extremamente atual e relevante no que tange a saúde do profissional.

Os achados deste estudo revelaram que a cultura de segurança do paciente na instituição é considerada adequada, uma vez que a maioria dos domínios apresentou um percentual entre $50 \%$ e $75 \%$ de positividade, mesmo assim mostra a necessidade de investimento para que se elevem esses percentuais. Os principais pontos de melhoria identificados foram a necessidade capacitações dos profissionais, seja por meio de estratégias e educação continuada ou permanente, aproximação dos gestores com a equipe multiprofissional por meio da comunicação assertiva, além da necessidade de avaliação do ambiente de trabalho, sobretudo o dimensionamento das equipes e os benefícios ofertados pelo serviço de saúde.

Dessa forma, compreende-se que o estudo possibilita o compartilhamento de informações relevantes e necessárias para as discussões relativas à segurança do paciente por meio da experiência da instituição hospitalar investigada. Os achados também colaboram com a criação e desenvolvimento de estratégias de melhoria na instituição pesquisada, sobretudo na articulação de uma comissão multidisciplinar para avaliação da segurança do paciente.

Sugere-se que outras pesquisas sobre o tema sejam realizadas, buscando identificar aspectos relevantes em outros serviços de saúde, possibilitando a realização de comparativos entre as práticas desenvolvidas e revisão nos modelos assistenciais e de gerenciamento disponíveis.

\section{Referências}

Alencar, S. V. G. L., do Nascimento, E. A., Brito, C. D., de Sousa Barbosa, D. D., de Andrade Marques, L., Luna, G. L. M., \& da Silva Probo, R. A. (2021). Segurança do paciente: representações sociais na visão dos profissionais da atenção primária à saúde. Revista Brasileira em Promoção da Saúde, $34: 11636$.

Barbosa, I. E. B., Fonseca, A. R., de Andrade, E. N. M., Maklouf, D. C., Ribeiro, M. C. S., da Silva Rodrigues, A. J. P., \& Sena Gomes, S. S. (2021). Segurança do paciente: principais eventos adversos na Unidade Terapia Intensiva. Revista Eletrônica Acervo Saúde, 13(2), e6454-e6454.

Carneiro, A. S., Dalmolin, G. D. L., Magnago, T. S. B. D. S., Moreira, L. P., Costa, E. D., \& Andolhe, R. (2021). Cultura de segurança do paciente em áreas críticas e não críticas: estudo comparativo. Revista da Escola de Enfermagem da USP, 55 e20210141.

Carvalho, R. D. L. (2011). Adaptação Transcultural do Safety Atitudes Questionarie para o Brasil - Questionário de Atitudes de Segurança. Tese (doutorado). Escola de Enfermagem de Ribeirão Preto, Universidade de São Paulo, Ribeirão preto, 2011.

Chauhan, R., Kaur, H., \& Chang, V. (2021). An Optimized Integrated Framework of Big Data Analytics Managing Security and Privacy in Healthcare Data. Wireless Personal Communications, 117(1).

Harada, M. D. J. C. S., Silva, A. E. B. D. C., Feldman, L. B., Tavares, S. S., Gerhardt, L. M., Lima, A. J. D., \& Kolankiewicz, A. C. B. (2021). Reflexões sobre sistemas de notificação de incidentes de segurança do paciente. Revista Brasileira de Enfermagem, $74 . \mathrm{e} 20200307$.

Jost, M. T., Branco, A., Araujo, B. R., Viegas, K., \& Caregnato, R. C. A. (2021). Ferramentas para a organização do processo de trabalho na segurança do paciente. Escola Anna Nery, 25(3).

Lima Neto, A. V., de Santana Silva, J. P., de Araújo, I. D. T., \& de Araújo Nunes, V. M. (2021). Produção científica em saúde coletiva pós-implantação do Programa Nacional de Segurança do Paciente. Revista Brasileira em Promoção da Saúde, 34.11671.

Lima, M. D. C. O., de Sales, C. I. S., de Oliveira Salvador, P. T. C., da Costa, T. D., \& Lopes, R. H. (2021). Perfil dos núcleos de segurança do paciente em serviços da Rede Cegonha no Nordeste brasileiro. Revista de Administração em Saúde, 21(84). e298.

Lopes, L. F. D. (2018). Métodos quantitativos aplicados ao comportamento organizacional [recurso eletrônico]. Santa Maria. Voix.

Madalozzo, M. M., Lucas, J. I. P., Kanan, L. A., Marcon, S. R. A., de Souza, A. S., Michelin, F. T., \& Hoffman, S. P. (2021). Cultura de segurança do paciente em um hospital acreditado de alta complexidade. Research, Society and Development, 10(6), e55510616113-e55510616113. 
Research, Society and Development, v. 10, n. 14, e326101422206, 2021

(CC BY 4.0) | ISSN 2525-3409 | DOI: http://dx.doi.org/10.33448/rsd-v10i14.22206

Mendes, L. A., Costa, A. C. L., Silva, D. C. Z., Simões, D. A. D. S., Côrrea, A. D. R., \& Manzo, B. F. (2021). Adesão da equipe de enfermagem às ações de segurança do paciente em unidades neonatais. Revista Brasileira de Enfermagem, 74. e20200765.

Nora, C. R. D., \& Junges, J. R. (2021). Segurança do paciente e aspectos éticos: revisão de escopo. Revista Bioética, 29, 304-316.

Nunes, D. A. A. (2021). Segurança do paciente cirúrgico em Rondônia. Saber Científico (1982-792X), 6(1),70-75.

Oliveira, A. D., \& Toledo, V. P. (2021). Segurança do paciente em uma unidade de internação psiquiátrica em hospital geral: estudo fenomenológico. Revista da Escola de Enfermagem da USP, 55.e03671.

Penariol, M. D. C. B., Pimentel, Á. B. N. M., Faria, É. T. S. S., Rodrigues, A. S., \& Milagres, C. S. (2021). Segurança do paciente no contexto da hemodiálise: uma revisão integrativa. Brazilian Journal of Health Review, 4(1),1620-1639.

Rocha, R. C., Abreu, I. M. D., Carvalho, R. E. F. L. D., Rocha, S. S. D., Madeira, M. Z. D. A., \& Avelino, F. V. S. D. (2021). Cultura de segurança do paciente em centros cirúrgicos: perspectivas da enfermagem. Revista da Escola de Enfermagem da USP, 55. e03774.

Rocha, R. C., Nunes, B. M. V. T., Araújo, A. A. C., Faria, L. F. L., \& Bezerra, M. A. R. (2021). Segurança do paciente na formação de técnicos de enfermagem. Revista Brasileira de Enfermagem, 75. e20201364.

Ruth, R. C. R., Bezerra, M. A. R., Martins, B. D. M. B., \& Nunes, B. M. V. T. (2021). Ensino da segurança do paciente na enfermagem: revisão integrativa. Enfermería Global, 20(4)700-743.

Santos, L. L., Camerini, F. G., Fassarella, C. S., Almeida, L. F. D., Setta, D. X. D. B., \& Radighieri, A. R. (2021). Medication time out como estratégia para a segurança do paciente: reduzindo erros de medicação. Revista Brasileira de Enfermagem, 74. e20200136.

Silva, B. J. R., Santos, B. D. V., de Andrade, C. R., Macedo, E. R., \& Andrade, H. S. (2021). Ações de enfermagem que promovem a segurança do paciente no âmbito hospitalar. Research, Society and Development, 10(5), e44110515202-e44110515202.

Siqueira, C. P., Figueiredo, K. C., Khalaf, D. K., Wall, M. L., Barbosa, S. D. F. F., \& Silva, T. A. (2021). Segurança do paciente em uma unidade de pronto atendimento: planejamento de ações estratégicas. Revista Enfermagem UERJ, 29, 55404.

Torrente, G., \& Barbosa, S. D. F. F. (2021). Instrumentos para avaliação da cultura de segurança do paciente nos serviços de emergência: revisão integrativa. Revista Brasileira de Enfermagem, 74. e190693.

Viana, K. E., Matsuda, L. M., Ferreira, A. M. D., Reis, G. A. X. D., Souza, V. S. D., \& Marcon, S. S. (2021). Cultura de segurança do paciente na ótica de profissionais de enfermagem. Texto \& Contexto-Enfermagem, 30:e20200219. 